\title{
Neutron-Antineutron Oscillations from Lattice QCD
}

\author{
Enrico Rinaldi, ${ }^{1,2, *}$ Sergey Syritsyn, ${ }^{1,3, \dagger}$ Michael L. Wagman, ${ }^{4, \$}$ Michael I. Buchoff, ${ }^{5}$ \\ Chris Schroeder, ${ }^{5}$ and Joseph Wasem ${ }^{5}$ \\ ${ }^{1}$ RIKEN BNL Research Center, Brookhaven National Laboratory, Upton, New York 11973, USA \\ ${ }^{2}$ Nuclear Science Division, Lawrence Berkeley National Laboratory, Berkeley, California 94720, USA \\ ${ }^{3}$ Department of Physics and Astronomy, Stony Brook University, Stony Brook, New York 11794, USA \\ ${ }^{4}$ Center for Theoretical Physics, Massachusetts Institute of Technology, Cambridge, Massachusetts 02139, USA \\ ${ }^{5}$ Lawrence Livermore National Laboratory, Livermore, California 94550, USA
}

(Received 15 September 2018; revised manuscript received 24 January 2019; published 22 April 2019)

\begin{abstract}
Fundamental symmetry tests of baryon number violation in low-energy experiments can probe beyond the standard model (BSM) explanations of the matter-antimatter asymmetry of the Universe. Neutronantineutron oscillations are predicted to be a signature of many baryogenesis mechanisms involving lowscale baryon number violation. This Letter presents first-principles calculations of neutron-antineutron matrix elements needed to accurately connect measurements of the neutron-antineutron oscillation rate to constraints on $|\Delta B|=2$ baryon number violation in BSM theories. Several important systematic uncertainties are controlled by using a state-of-the-art lattice gauge field ensemble with physical quark masses and approximate chiral symmetry, performing nonperturbative renormalization with perturbative matching to the modified minimal subtraction scheme, and studying excited state effects in two-state fits. Phenomenological implications are highlighted by comparing expected bounds from proposed neutronantineutron oscillation experiments to predictions of a specific model of postsphaleron baryogenesis. Quantum chromodynamics is found to predict at least an order of magnitude more events in neutronantineutron oscillation experiments than previous estimates based on the "MIT bag model" for fixed BSM parameters. Lattice artifacts and other systematic uncertainties that are not controlled in this pioneering calculation are not expected to significantly change this conclusion.
\end{abstract}

DOI: 10.1103/PhysRevLett.122.162001

Introduction.-Beyond the standard model (BSM) violation of baryon number conservation is necessary to explain the observed matter-antimatter asymmetry of the Universe. Baryogenesis explanations involving physics at high scales, such as leptogenesis, are appealing but difficult to test in low-energy experiments. Alternative explanations such as postsphaleron baryogenesis involve low-scale baryon number violation that can be directly constrained by current and near-future experiments. Extracting robust BSM theory constraints from these experiments is critical for fundamental symmetry tests of baryon and lepton number violation addressing the long-standing mystery of matter-antimatter asymmetry.

Neutron-antineutron oscillations $(n-\bar{n})$ are predicted as a signature of low-scale baryogenesis in BSM theories including $S O(10)$ grand unified theories, left-right symmetric theories, $R$-parity-violating supersymmetry, low-scale

Published by the American Physical Society under the terms of the Creative Commons Attribution 4.0 International license. Further distribution of this work must maintain attribution to the author(s) and the published article's title, journal citation, and DOI. Funded by SCOAP . quantum gravity, extradimensional theories, and string theories with exotic instantons; see Refs. [1-3] for comprehensive reviews and further references. Experimental constraints on $n-\bar{n}$ come from large underground detection experiments such as super-K [4] and SNO [5] or from cold neutron time-of-flight experiments [6]. Results are presented as bounds on the neutron-antineutron oscillation time $\tau_{n-\bar{n}}$ governing the time-dependent probability $P_{n-\bar{n}}=$ $\sin ^{2}\left(t / \tau_{n-\bar{n}}\right)$ for a free neutron in vacuum to turn into an antineutron [3].

The best direct bound on $\tau_{n-\bar{n}}$ is from the cold neutron experiment at Institut Laue-Langevin (ILL), $\tau_{n-\bar{n}}>0.89 \times$ $10^{8} \mathrm{~s}$ [6], which is essentially background free and can be improved with larger neutron flux, magnetic shielding, and the latest technologies in neutron optics. There has been a proposal for a new cold neutron experiment at the European spallation source (ESS) that would be $\approx 10^{3}$ times more sensitive than the ILL experiment and improve cold neutron constraints on $\tau_{n-\bar{n}}$ by a factor of 32 [7,8], as well as experiments at other reactors [9]. The best bound to date on the neutron-antineutron transition time within oxygen-16 is from super-K, $\tau_{O^{16}}>1.9 \times 10^{32}$ years [4], which constrains the free oscillation time $\tau_{n-\bar{n}}>2.7 \times 10^{8} \mathrm{~s}$ after 
taking into account nuclear structure effects [10]. In the future, we expect underground neutrino facilities like DUNE to be able to provide competitive bounds thanks to improved background-rejection techniques [11].

BSM theories cannot directly predict $\tau_{n-\bar{n}}$, which also depends on low-energy dynamics of quantum chromodynamics (QCD) responsible for binding quarks into hadrons. Because BSM and QCD effects are important at different scales, any BSM mechanism mediating $n-\bar{n}$ oscillations can be summarized into a particular linear combination of effective six-quark operators violating baryon number by two units. Their matrix elements between neutron and antineutron states are determined by nonperturbative QCD and have to be computed before any BSM predictions for $\tau_{n-\bar{n}}$ can be made. Prior to this work, only "MIT bag model" estimates of these six-quark matrix elements $[12,13]$ have been available and connections between $\tau_{n-\bar{n}}$ and new physics constraints have included unknown model uncertainties.

The purpose of this Letter is to present a lattice QCD (LQCD) calculation of these six-quark matrix elements and to discuss its impact on possible discovery of new physics and theories of baryogenesis. Our work improves upon preliminary LQCD results [14] by using chiral quarks at the physical point and robust nonperturbative renormalization, and upon recent results [15] by analyzing excited state effects. With most of LQCD uncertainties under control, we find that QCD predicts 1-2 orders of magnitude larger rate of $n-\bar{n}$ oscillations than previously expected. Our results have recently been presented [16] at workshops dedicated to devising a plan to observe $n-\bar{n}$ oscillations at the ESS, future reactors, and underground laboratories [17], and used in generic effective field theories (EFTs) for baryogenesis such as the recent work in Ref. [18].

Neutron-antineutron operators.-The basis for the lowest-dimension operators for $n-\bar{n}$ transitions that are color singlets and electrically neutral was constructed in Refs. [12,13,19-21]

$$
\begin{aligned}
& \mathcal{O}_{\chi_{1} \chi_{2} \chi_{3}}^{1}=\left(u^{T} C P_{\chi_{1}} u\right)\left(d^{T} C P_{\chi_{2}} d\right)\left(d^{T} C P_{\chi_{3}} d\right) T^{S S S}, \\
& \mathcal{O}_{\chi_{1} \chi_{2} \chi_{3}}^{2}=\left(u^{T} C P_{\chi_{1}} d\right)\left(u^{T} C P_{\chi_{2}} d\right)\left(d^{T} C P_{\chi_{3}} d\right) T^{S S S}, \\
& \mathcal{O}_{\chi_{1} \chi_{2} \chi_{3}}^{3}=\left(u^{T} C P_{\chi_{1}} d\right)\left(u^{T} C P_{\chi_{2}} d\right)\left(d^{T} C P_{\chi_{3}} d\right) T^{A A S},
\end{aligned}
$$

where quark spin indices are implicitly contracted in the parentheses and quark color indices are implicitly contracted with the tensors

$$
\begin{aligned}
T_{\{i j\}\{k l\}\{m n\}}^{S S S} & =\varepsilon_{i k m} \varepsilon_{j l n}+\varepsilon_{j k m} \varepsilon_{i l n}+\varepsilon_{i l m} \varepsilon_{j k n}+\varepsilon_{i k n} \varepsilon_{j l m}, \\
T_{[i j][k l]\{m n\}}^{A A S} & =\varepsilon_{i j m} \varepsilon_{k l n}+\varepsilon_{i j n} \varepsilon_{k l m},
\end{aligned}
$$

$P_{L, R}=\frac{1}{2}\left(1 \mp \gamma_{5}\right)$ are chiral projectors, and the Euclidean charge conjugation matrix $C$ satisfies $C \gamma_{\mu} C^{\dagger}=-\gamma_{\mu}^{T}$. Symmetries together with the Fierz relation $\mathcal{O}_{\chi \chi \chi^{\prime}}^{2}-$ $\mathcal{O}_{\chi \chi \chi^{\prime}}^{1}=3 \mathcal{O}_{\chi \chi \chi^{\prime}}^{3}$ reduce the number of independent operators to 14 ( $7+7$ related by parity). In this Letter, we use basis operators from chiral isospin multiplets that renormalize multiplicatively $[15,22]$ and have particular implications for phenomenology. An extended discussion of the operators and their chiral properties is reported in Ref. [23]. There are three standard model (SM) gaugesinglet operators (Two additional operators arise in dimensional regularization at two-loop order [22] that are exactly equal to $Q_{1,3}$ in lattice regularization by Fierz identities. These Fierz identities are preserved in the modified minimal subtraction $(\overline{\mathrm{MS}})$ scheme if one-loop matching is consistently included with two-loop running. At high scales $\Lambda_{\mathrm{BSM}} \gg \Lambda_{\mathrm{QCD}}$ Fierz identity violations can be neglected even if BSM matching is performed at tree level.) that provide the dominant contributions to $n-\bar{n}$ transitions in SM EFT,

$$
\begin{aligned}
& Q_{1}=-4\left(u^{T} C P_{R} d\right)\left(u^{T} C P_{R} d\right)\left(d^{T} C P_{R} d\right) T^{A A S}, \\
& Q_{2}=-4\left(u^{T} C P_{L} d\right)\left(u^{T} C P_{R} d\right)\left(d^{T} C P_{R} d\right) T^{A A S}, \\
& Q_{3}=-4\left(u^{T} C P_{L} d\right)\left(u^{T} C P_{L} d\right)\left(d^{T} C P_{R} d\right) T^{A A S} .
\end{aligned}
$$

The fourth $S U(2)_{L} \times U(1)$ singlet operator

$$
\begin{aligned}
Q_{4}= & -\frac{4}{5}\left(u^{T} C P_{R} u\right)\left(d^{T} C P_{R} d\right)\left(d^{T} C P_{R} d\right) T^{S S S} \\
& -\frac{16}{5}\left(u^{T} C P_{R} d\right)\left(u^{T} C P_{R} d\right)\left(d^{T} C P_{R} d\right) T^{S S S}
\end{aligned}
$$

has vanishing matrix elements in the $S U(2)$ isospin limit and is not studied in this Letter. Isospin-violating corrections provide a subdominant systematic uncertainty and are also neglected. $S U(2)_{L}$-nonsinglet operators can also lead to $n-\bar{n}$ oscillations

$$
\begin{aligned}
Q_{5}= & \left(u^{T} C P_{R} u\right)\left(d^{T} C P_{L} d\right)\left(d^{T} C P_{L} d\right) T^{S S S}, \\
Q_{6}= & -4\left(u^{T} C P_{R} d\right)\left(u^{T} C P_{L} d\right)\left(d^{T} C P_{L} d\right) T^{S S S}, \\
Q_{7}= & -\frac{4}{3}\left(u^{T} C P_{L} u\right)\left(d^{T} C P_{L} d\right)\left(d^{T} C P_{R} d\right) T^{S S S} \\
& -\frac{8}{3}\left(u^{T} C P_{L} d\right)\left(u^{T} C P_{L} d\right)\left(d^{T} C P_{R} d\right) T^{S S S} .
\end{aligned}
$$

These operators transform in the same chiral irreducible representation but describe different chiral multiplet components and do not mix under renormalization [22,23]. Isospin $S U(2)$ symmetry leads to the following relation between matrix elements

$$
\left\langle\bar{n}\left|Q_{5}\right| n\right\rangle=\left\langle\bar{n}\left|Q_{6}\right| n\right\rangle=-\frac{3}{2}\left\langle\bar{n}\left|Q_{7}\right| n\right\rangle .
$$

The complete chiral basis of QCD and quantum electrodynamics singlet operators is given by $Q_{I}, Q_{I}^{P}, I=1 \ldots 7$ 
where parity-transformed operators are $Q_{I}^{P}=\left.\left(-Q_{I}\right)\right|_{L \leftrightarrow R}$. The operators (2)-(4) are related to the ones in Eq. (1) as

$Q_{1}=-4 \mathcal{O}_{R R R}^{3}, \quad Q_{2}=-4 \mathcal{O}_{L R R}^{3}, \quad Q_{3}=-4 \mathcal{O}_{L L R}^{3}$,

$Q_{4}=-\frac{4}{5} \mathcal{O}_{R R R}^{1}-\frac{16}{5} \mathcal{O}_{R R R}^{2}, \quad Q_{5}=\mathcal{O}_{R L L}^{1}$,

$Q_{6}=-4 \mathcal{O}_{R L L}^{2}, \quad Q_{7}=-\frac{4}{3} \mathcal{O}_{L L R}^{1}-\frac{8}{3} \mathcal{O}_{L L R}^{2}$.

Because of symmetries and Eq. (5), only four separate nucleon matrix elements $\left\langle\bar{n}\left|Q_{1,2,3,5}\right| n\right\rangle$ need to be determined using lattice QCD methods.

Lattice QCD results.-For this calculation, we use an ensemble of QCD gauge field configurations on a $48^{3} \times 96$ lattice generated with Iwasaki gauge action and $N_{f}=$ $2+1$ flavors of dynamical Möbius Domain Wall fermions with masses almost exactly at the physical point [24]. The pion mass is $m_{\pi}=139.2(4) \mathrm{MeV}$ and the lattice spacing is $a=0.1141(3) \mathrm{fm}$. With the physical lattice size $L \approx 5.45 \mathrm{fm}$, and $m_{\pi} L=3.86$, finite volume effects on the matrix elements are estimated from chiral perturbation theory to be $\lesssim 1 \%$ [25]. Discretization effects are expected to be small because the meson decay constants $f_{\pi, K}$ [24], the nucleon mass and dispersion [26] on this ensemble are very close to physical.

We calculate lattice correlation functions on 30 independent gauge field configurations separated by $40 \mathrm{MD}$ steps using point-source quark propagators aided by allmode-averaging sampling [27] to reduce stochastic uncertainty. On each configuration, we compute 1 exact and 81 low-precision samples evenly distributed over the fourdimensional volume. For the latter, quark propagators are computed with low-mode deflation and 250 iterations of the conjugate gradient algorithm. The propagators are contracted into intermediate baryon blocks $[28,29]$ representing (anti)neutron source or sink operators made of point and Gaussian-smeared quarks and denoted by $n^{J=P, S}$, respectively. These blocks are finally contracted into (anti)neutron two-point correlation functions of $P$ source and $J=P, S$ sink operators

$G_{2 p t}^{P J}(t)=\sum_{\mathbf{x}}\left\langle n_{\uparrow}^{J}(\mathbf{x}, t) \bar{n}_{\uparrow}^{P}(0)\right\rangle=\sum_{\mathfrak{n}} \sqrt{Z_{\mathfrak{n}}^{J} Z_{\mathfrak{n}}^{P}} e^{-E_{\mathfrak{n}} t}$,

as well as three-point correlation functions involving $J=S, P$ antineutron sources, neutron sinks, and sixantiquark operators $\bar{Q}_{I}$ that are obtained from $Q_{I}$ by charge conjugation and have identical matrix elements,

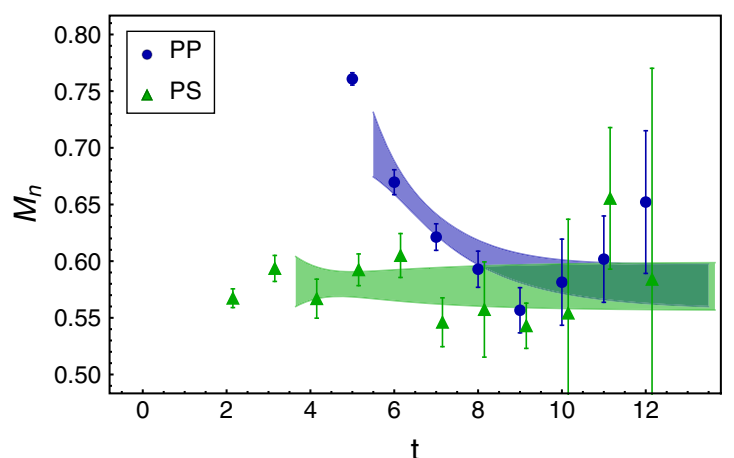

FIG. 1. Neutron effective mass $M_{n}^{P J}(t)=\ln \left[G_{2 p t}^{P J}(t) / G_{2 p t}^{P J}(t+a)\right]$ determined from point-point and point-smeared $(J=P, S)$ twopoint correlation functions. Lattice data points are shifted for visibility and compared to two-state fits (shaded bands). The asymptotic $t$ result is compatible with the nucleon mass when converted to physical units, indicating negligible discretization and finite volume effects.

$$
\begin{aligned}
G_{3 p t}^{J J^{\prime}}\left(\tau, t ; Q_{I}\right) & =\sum_{\mathbf{x}, \mathbf{y}}\left\langle n_{\uparrow}^{J}(\mathbf{x}, t-\tau) \bar{Q}_{I}(0) n_{\downarrow}^{J^{\prime}}(\mathbf{y},-\tau)\right\rangle \\
& =\sum_{\mathfrak{n}, \mathfrak{m}} \sqrt{Z_{\mathfrak{n}}^{J} Z_{\mathfrak{m}}^{J^{\prime}}} e^{-E_{\mathfrak{n}}(t-\tau)} e^{-E_{\mathfrak{m}} \tau}\left\langle\overline{\mathfrak{n}}, \uparrow\left|Q_{I}\right| \mathfrak{m}, \uparrow\right\rangle,
\end{aligned}
$$

where $|\mathfrak{m}, \uparrow\rangle(|\overline{\mathfrak{n}}, \uparrow\rangle)$ denote the spin-up (anti)neutron states, $(\tau, t)$ are the Euclidean time intervals from the source to the operator and the sink, respectively.

The $n-\bar{n}$ ground-state matrix elements are extracted with two-state fits using optimal shrinkage [30], variable projection (VarPro) [31], and weighted averages of results for a variety of source or sink separations that are described at length in our companion paper [23]. First, $G_{2 p t}^{P P}$ and $G_{2 p t}^{P S}$ are fitted in order to determine $E_{0,1}$ and $\sqrt{Z_{0}^{P, S}}$. An example fit is shown in Fig. 1. These results are subsequently used to extract matrix element results from linear fits to $G_{3 p t}^{S S}$ and $G_{3 p t}^{P S}+G_{3 p t}^{S P}$. Ten different source or sink separations are included in order to isolate and remove excited state effects. Ratios of $G_{3 p t}^{J J^{\prime}}$ to $G_{2 p t}^{J J^{\prime}}$ for operator $Q_{2}$, that reach a plateau when the ground state saturates the correlation functions and excited state contributions have become negligible, are shown in Fig. 2 including fit results and statistical uncertainties.

In regularization schemes which do not violate chiral symmetry, operator mixing between $Q_{I}^{(P)}(2)-(4)$ is forbidden, as in the continuum perturbation theory calculation of Ref. [22]. Because quark mass, residual chiral symmetry breaking, and nonperturbative effects might lead to operator mixing on a lattice, we compute the renormalization and mixing of these operators nonperturbatively using the regularization-invariant-momentum (RI-MOM) scheme [32]. RI-MOM renormalization factors $Z_{I J}^{\mathrm{RI}}, Q_{I}^{\mathrm{RI}}=$ $Z_{I J}^{\mathrm{RI}} Q_{J}^{\text {bare }}$ at momentum $p$ are defined as 

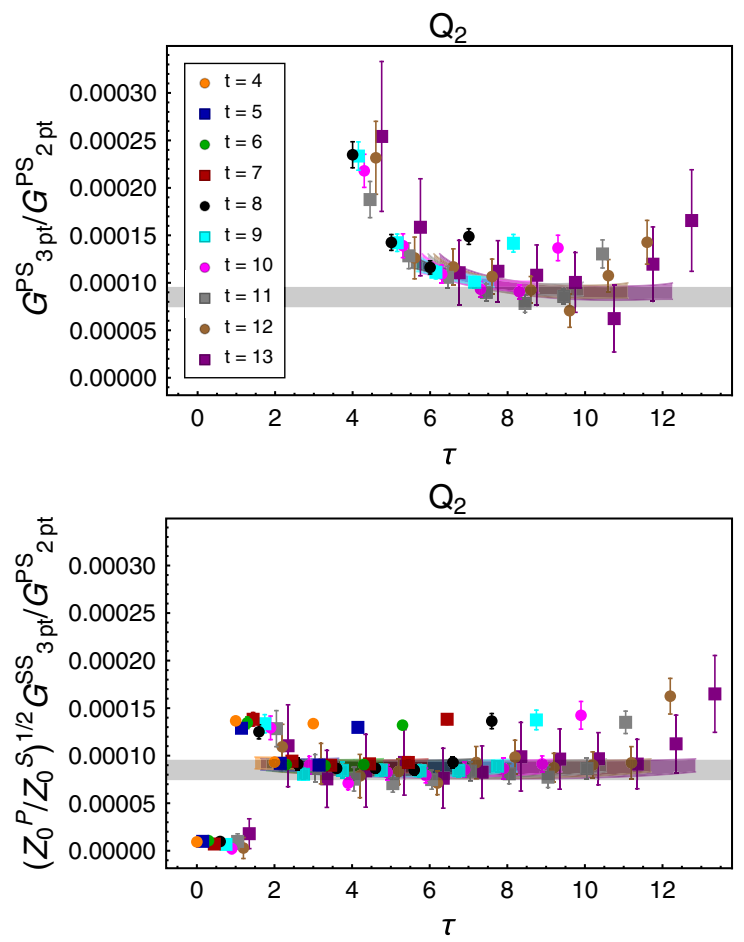

FIG. 2. Ratios of three-point correlation functions for operator $Q_{2}$ to two-point functions vs operator insertion time $\tau$ and 10 different source or sink separations $t$. Lattice data points are shifted for visibility. These data are compared to two-state fits (colored shaded bands) used to extract the ground state bare matrix elements, which are shown with statistical uncertainty for a particular value of fit ranges (horizontal bands).

$$
\left[Z_{q}^{\mathrm{RI}}(p)\right]^{-3} Z_{I J}^{\mathrm{RI}}(p) \Lambda_{J K}(p)=\delta_{I K},
$$

where $Z_{q}^{\mathrm{RI}}$ is the quark field renormalization and $\Lambda_{J K}(p)$ are amputated Green's functions of the lattice operators $Q_{J}$ and quark fields carrying momenta $\pm p$ projected onto the spin-color-flavor structure of $Q_{K}$. All steps to calculate the renormalization factors numerically can be found in the companion paper [23]. We find that the matrices $\Lambda_{I J}$ and therefore $Z_{I J}^{\mathrm{RI}}$ are diagonal in the chiral basis (2)-(4) up to $O\left(10^{-3}\right)$ [23], thanks to chiral symmetry of the lattice action we use. We neglect this residual mixing and identify $Z_{I}=Z_{I I}$ below.

The scale-independent combinations

$$
Z_{I}^{\mathrm{SI}}\left(\mu_{0}, p\right)=Z_{I}^{\mathrm{RI}}(p)\left(\frac{Z_{I}^{\mathrm{RI}}\left(\mu_{0}\right)}{Z_{I}^{\mathrm{RI}}(|p|)}\right)^{\text {pert }}
$$

of perturbative and nonperturbative factors have residual dependence on the lattice momentum $p_{\nu}$ due to nonperturbative effects $\propto p^{-2}$, discretization effects $\propto(a p)^{2}$, and rotational symmetry breaking. We analyze these effects following Ref. [33], with a representative fit for operator $Q_{2}$ and $\mu_{0}=2 \mathrm{GeV}$ shown in Fig. 3. Fits with varying momentum ranges up to $1.6 \leq p \leq 4.5 \mathrm{GeV}$ are used to

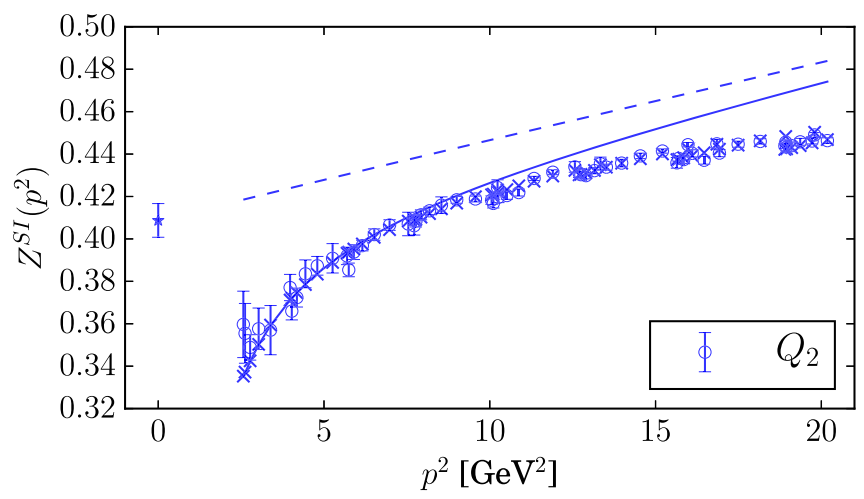

FIG. 3. RI-MOM "scale-independent" renormalization factors $Z^{\text {SI }}$ for the operator $Q_{2}$. Lattice data (circles) are fit to a constant (star) plus lattice artifacts: including $\propto(a p)^{2}$ (dashed line), $\propto(a p)^{-2}$ (solid line), and $O(4)$-breaking (crosses) terms [33].

define central values and stochastic and systematic uncertainties for $Z_{I}^{\mathrm{RI}}$. We have found no substantial difference between fits using one- and two-loop perturbative factors in Eq. (10). Fit details and similar plots for other operators can be found in Ref. [23]. RI-MOM matrix element results are then converted to the $\overline{\mathrm{MS}}(2 \mathrm{GeV})$ and $\overline{\mathrm{MS}}(700 \mathrm{TeV})$ scheme using one-loop matching [22]

$$
\left\langle\bar{n}\left|Q_{I}^{\overline{\mathrm{MS}}}\right| n\right\rangle=\left[\frac{Z_{I, N_{f}}^{\overline{\mathrm{MS}}}=4}{Z_{I, N_{f}=3}^{\mathrm{RI}}}\right]^{\mathrm{pert}} Z_{I, N_{f}=3}^{\mathrm{RI}}\left\langle\bar{n}\left|Q_{1}\right| n\right\rangle,
$$

where the difference between $N_{f}=3$ and $N_{f}=4$ QCD is taken into account by matching $\alpha_{S}$ [34] and operator normalization at the charm quark threshold $\mu=M_{c}$. Statistical and systematic uncertainties of the renormalization factors and the bare matrix elements are added in quadrature. Higher-order matching uncertainties are estimated to be $\lesssim 7 \%$ based on the size of one-loop matching effects [22] and are neglected. Final results for the $\overline{\mathrm{MS}}$ matrix elements are shown in Table I and compared to previous MIT bag model results.

Phenomenological implications.-In BSM theories where $\Delta B=2$ transitions are permitted, experimentally observable $n-\bar{n}$ oscillations are low-energy phenomena that can be described in an EFT containing only SM fields. The low-energy EFT will include $\Delta B=2$ terms involving the operators $Q_{I}$ above

$$
\mathcal{L}_{n-\bar{n}}=\sum_{I=1}^{7}\left[C_{I}(\mu) Q_{I}(\mu)+C_{I}^{P}(\mu) Q_{I}^{P}(\mu)\right],
$$

where the $C_{I}$ are numerical coefficients with mass dimension $(-5)$ that are predicted to be nonzero in some BSM theories. The $S U(2)_{L}$-singlet operators are EW symmetric and their coefficients should scale as $C_{1,2,3,4} \sim \Lambda_{\mathrm{BSM}}^{-5}$ in naive dimensional analysis. In contrast, the $S U(2)_{L}$ 
TABLE I. For each operator $Q_{I}$ we show its renormalized matrix element value $\mathcal{M}_{I}^{\overline{\mathrm{MS}}}$ in units of $\left[10^{-5} \mathrm{GeV}^{6}\right]$. The total uncertainty includes statistical and systematic errors added in quadrature. Renormalized results are obtained through nonperturbative RI-MOM renormalization and perturbative matching to $\overline{\mathrm{MS}}$ at two scales: $2 \mathrm{GeV}$ and $700 \mathrm{TeV}$ in column two and three, respectively. The last two columns show a comparison between the lattice QCD matrix elements and the results of the same matrix elements for two choices of the MIT bag model from Ref. [12].

\begin{tabular}{lcccc}
\hline \hline Operator & $\mathcal{M}_{I}^{\overline{\mathrm{MS}}}(2 \mathrm{GeV})$ & $\mathcal{M}_{I}^{\overline{\mathrm{MS}}}(700 \mathrm{TeV})$ & $\mathcal{M}_{I}^{\overline{\mathrm{MS}}} / \mathrm{MIT}$ bag A $(2 \mathrm{GeV})$ & $\mathcal{M}_{I}^{\overline{\mathrm{MS}}} / \mathrm{MIT}$ bag B $(2 \mathrm{GeV})$ \\
\hline$Q_{1}$ & $-46(13)$ & $-26(7)$ & 4.2 & 5.2 \\
$Q_{2}$ & $95(17)$ & $144(26)$ & 7.5 & 8.7 \\
$Q_{3}$ & $-50(12)$ & $-47(11)$ & 5.1 & 6.1 \\
$Q_{5}$ & $-1.06(48)$ & $-0.23(10)$ & -0.8 & 1.6 \\
\hline \hline
\end{tabular}

nonsinglet operators $Q_{1, \ldots, 7}^{P}$ and $Q_{5,6,7}^{(P)}$ can only appear in an SM gauge-invariant Lagrangian in products with additional SM Higgs (or BSM) fields to make them $S U(2)_{L}$ singlets. Assuming the former, their coefficients should scale as $C_{1, \ldots, 7}^{P}\left(\Lambda_{\mathrm{BSM}}\right) \sim v^{2} \Lambda_{\mathrm{BSM}}^{-7}$ and $C_{5,6,7} \sim v^{4} \Lambda_{\mathrm{BSM}}^{-9}$, where $v$ is the vacuum expectation value of the Higgs field. For $v \ll \Lambda_{\mathrm{BSM}}$, this provides a significant additional suppression on $n-\bar{n}$ oscillation rate contributions from $S U(2)_{L}$-nonsinglet operators.

The $n-\bar{n}$ oscillation rate is given by the matrix element of the associated Hamiltonian between neutron and antineutron states, which in the isospin limit of QCD simplifies to

$$
\tau_{n-\bar{n}}^{-1}=\left|\sum_{I=1,2,3,5} \hat{C}_{I}(\mu)\left\langle\bar{n}\left|Q_{I}(\mu)\right| n\right\rangle\right|,
$$

where $\quad \hat{C}_{I}=C_{I}-C_{I}^{P} \quad$ for $\quad I=1, \ldots, 4 \quad$ and $\quad \hat{C}_{5}=$ $\left(C_{5}-C_{5}^{P}\right)+\left(C_{6}-C_{6}^{P}\right)-\frac{2}{3}\left(C_{7}-C_{7}^{P}\right)$. Contributions involving $C_{4}^{(P)}$ vanish exactly in the isospin limit considered here, although in principle isospin-violating $C_{4}^{(P)}$ contributions could play a role in particular BSM models. Using the LQCD results above, the $n-\bar{n}$ oscillation rate is given by

$$
\begin{aligned}
\tau_{n-\bar{n}}^{-1}= & \frac{10^{-9} \mathrm{~s}^{-1}}{(700 \mathrm{TeV})^{-5}} \mid 4.2(1.1) \hat{C}_{1}^{\overline{\mathrm{MS}}}(\mu)-8.6(1.5) \hat{C}_{2}^{\overline{\mathrm{MS}}}(\mu) \\
& +4.5(1.1) \hat{C}_{3}^{\overline{\mathrm{MS}}}(\mu)+\left.0.096(43) \hat{C}_{5}^{\overline{\mathrm{MS}}}(\mu)\right|_{\mu=2 \mathrm{GeV}}
\end{aligned}
$$

It is noteworthy that, in addition to the $\left(v / \Lambda_{\mathrm{BSM}}\right)^{2}$ suppression, contributions from $\hat{C}_{5}$ are suppressed by almost 2 orders of magnitude compared to those from $\hat{C}_{1,2,3}$ due to the relative sizes of the associated QCD matrix elements computed here.

Predictions for nonzero $\tau_{n-\bar{n}}^{-1}$ arise in some BSM theories explaining the matter-antimatter asymmetry of the Universe and other outstanding problems of the SM and cosmology. An example is provided by $n-\bar{n}$ oscillations in left-right symmetric gauge theories where the SM gauge group is embedded in $S U(2)_{L} \times S U(2)_{R} \times S U(4)_{C}$ with $(B-L)$ acting as a fourth color [35-37]. Postsphaleron baryogenesis occurs after a colored BSM scalar field develops a $(B+L)$-breaking vacuum expectation value that leads to Majorana neutrino masses and $n-\bar{n}$ oscillations. The $n-\bar{n}$ oscillation rate in this model only involves $Q_{1}$ at tree level and $\tau_{n-\bar{n}}^{-1}$ is given by the first term in Eq. (14). The reach of current and future $n-\bar{n}$ oscillation experiments into the parameter space of this $S U(2)_{L} \times S U(2)_{R} \times S U(4)_{C}$ model is four to five times higher according to LQCD than the bag model. In turn, because oscillation probabilities are $\propto \tau_{n-\bar{n}}^{-2}$ [3], this model would predict 16 to 25 times larger number of observed events in quasifree neutron experiments. Updated phenomenological studies of this and other BSM theories of low-scale baryogenesis are needed to determine the reach of current and future $n-\bar{n}$ experiments into BSM parameter space.

Conclusions.-An LQCD calculation of six-quark matrix elements is presented that provides, for the first time, renormalized $n-\bar{n}$ transition matrix elements in the $\overline{\mathrm{MS}}$ scheme at $2 \mathrm{GeV}$. These renormalized results with welldefined scale dependence are required to reliably connect experimental measurements of $\tau_{n-\bar{n}}$ to the baryon numberviolating new physics scale $\Lambda_{\mathrm{BSM}}$.

This calculation is performed with physical pion masses, a chirally-symmetric fermion discretization, and a large spacetime volume. Ground-state matrix elements are extracted using two-state fits and systematic uncertainties associated with excited states are estimated through variation of the fitting region. Finite volume effects are predicted by chiral EFT to be $\lesssim 1 \%$ [25] for the $L \approx 5.45 \mathrm{fm}$ volume used in this study. Even though we only use one lattice spacing $a \approx 0.114 \mathrm{fm}$, discretization effects are expected to be small because of automatic $\mathcal{O}(a)$ improvement due to the chiral symmetry of the DWF action. Additional systematic effects due to the uncertainty in the lattice scale $\delta a / a \approx 1.3 \%$ are negligible. The error budget of our final results is dominated by limited statistics. Statistical uncertainties and systematic uncertainties associated with discretization and finite volume effects can be improved in the future using additional ensembles [24]. However, they are 
very unlikely to change the dramatic impact that $\mathrm{QCD}$ effects have on these six-quark matrix elements.

To conclude, there has been recent phenomenological interest in $n-\bar{n}$ oscillations and the possibility of new searches for $n-\bar{n}$ vacuum oscillations and transitions in nuclei at ESS, DUNE, and other experiments [7-9,11]. The magnitudes of electroweak-singlet $n-\bar{n}$ transition matrix elements are four to eight times larger than those computed in the MIT bag model [12]. Experiments should consequently observe 16-64 times more neutron-antineutron oscillation events for fixed BSM physics parameters. Future searches for $n-\bar{n}$ oscillations will be able to probe the parameter space of several viable baryogenesis scenarios [18]. Our results, despite being pioneering, indicate that experimental searches of $n-\bar{n}$ transitions are about $1-2$ orders of magnitude more sensitive to baryon number violating interactions in BSM physics than previously expected and will be able to put more stringent constraints on various baryogenesis mechanisms.

We are indebted to Norman Christ, Bob Mawhinney, Taku Izubuchi, Oliver Witzel, and the rest of the RBC/ UKQCD collaboration for access to the physical point, domain-wall lattices, and propagators used in this Letter. We would like to thank Yuri Kamyshkov, Rabi Mohapatra, Martin Savage, Steve Sharpe, Robert Shrock, Mike Snow, Brian Tiburzi, and hosts of others for discussions over the duration of this project. Computing support for this Letter was provided in part from the LLNL Institutional Computing Grand Challenge program and from the USQCD Collaboration, which is funded by the Office of Science of the U.S. Department of Energy. This research used resources of the Argonne Leadership Computing Facility, which is a DOE Office of Science User Facility supported under Contract No. DE-AC02-06CH11357. This work has been supported by the U.S. Department of Energy under Awards No. DE-AC52-07NA27344 (Lawrence Livermore National Laboratory) and No. DE-FG0200ER41132 (Institute for Nuclear Theory). Brookhaven National Laboratory is supported by the U.S. Department of Energy under Award No. DE-SC0012704. S. S. acknowledges support by the Relativistic Heavy Ion Collider Physics Fellow Program of the RIKEN BNL Research Center. E. R. is supported by the RIKEN Special Postdoctoral Researcher Fellowship. M. L. W. was supported by an MIT Pappalardo Fellowship and acknowledges support by the U.S. Department of Energy, Office of Science, Office of Nuclear Physics under Award No. DE-SC0011090.

*erinaldi@bnl.gov

†sergey.syritsyn@stonybrook.edu

*mlwagman@mit.edu

[1] R. N. Mohapatra, Neutron-anti-neutron oscillation: Theory and phenomenology, J. Phys. G 36, 104006 (2009).
[2] K. Babu et al., Neutron-antineutron oscillations: A snowmass 2013 white paper, arXiv:1310.8593.

[3] D. G. Phillips et al., Neutron-antineutron oscillations: Theoretical status and experimental prospects, Phys. Rep. 612, 1 (2016).

[4] K. Abe et al. (Super-Kamiokande Collaboration), Search for n-nbar oscillation in Super-Kamiokande, Phys. Rev. D 91, 072006 (2015).

[5] B. Aharmim et al. (SNO Collaboration), The search for neutron-antineutron oscillations at the Sudbury Neutrino Observatory, Phys. Rev. D 96, 092005 (2017).

[6] M. Baldo-Ceolin et al., A new experimental limit on neutron-antineutron oscillations, Z. Phys. C 63, 409 (1994).

[7] D. Milstead, A new high sensitivity search for neutronantineutron oscillations at the ESS, arXiv:1510.01569.

[8] M. J. Frost, The NNbar experiment at the European spallation source, arXiv:1607.07271.

[9] A. K. Fomin, A. P. Serebrov, O. M. Zherebtsov, M. E. Chaikovskii, A. N. Murashkin, E. N. Leonova, O.P. Fedorova, V. G. Ivochkin, V. A. Lyamkin, D. V. Prudnikov, and A. V. Chechkin, Project of NNbar experiment at the WWR-M reactor, KnE Energy 3, 109 (2018).

[10] E. Friedman and A. Gal, Realistic calculations of nuclear disappearance lifetimes induced by $\mathrm{n}$ anti-n oscillations, Phys. Rev. D 78, 016002 (2008).

[11] J.E. T. Hewes, Searches for bound neutron-antineutron oscillation in liquid argon time projection chambers, Technical Report FERMILAB-THESIS-2017-271662648, Fermi National Accelerator Laboratory (FNAL), Batavia, IL, 2017.

[12] S. Rao and R. Shrock, n nbar transition operators and their matrix elements in the MIT bag model, Phys. Lett. 116B, 238 (1982).

[13] R. Sumathi and R. E. Shrock, Six-fermion (BL)-violating operators of arbitrary generational structure, Nucl. Phys. B232, 143 (1984).

[14] M. Buchoff, C. Schroeder, and J. Wasem, Neutronantineutron oscillations on the lattice, in Proceedings of the 30th International Symposium on Lattice Field Theory-PoS (Lattice 2012) (Sissa Medialab, Trieste, Italy, 2012), p. 128.

[15] S. Syritsyn, M. I. Buchoff, C. Schroeder, and J. Wasem, Neutron-antineutron oscillation matrix elements with domain wall fermions at the physical point, in Proceedings of the 33rd International Symposium on Lattice Field Theory-PoS(LATTICE 2015) (Sissa Medialab, Trieste, Italy, 2016), Vol. 132, p. 132.

[16] E. Rinaldi, Lattice calculations for neutron-antineutron oscillations, Zenodo: http://doi.org/10.5281/zenodo.2539735.

[17] A. Young et al., Particle physics with neutrons at ESS (to be published)

[18] C. Grojean, B. Shakya, J. D. Wells, and Z. Zhang, Implications of an improved neutron-antineutron oscillation search for baryogenesis: A minimal effective theory analysis, Phys. Rev. Lett. 121, 171801 (2018).

[19] L. N. Chang and N. P. Chang, B-L nonconservation and neutron oscillation, Phys. Lett. 92B, 103 (1980).

[20] T.-K. Kuo and S. T. Love, Neutron oscillations and the existence of massive neutral leptons, Phys. Rev. Lett. 45, 93 (1980). 
[21] W. E. Caswell, J. Milutinovic, and G. Senjanovic, Matterantimatter transition operators: A manual for modeling, Phys. Lett. 122B, 373 (1983).

[22] M. I. Buchoff and M. Wagman, Perturbative renormalization of neutron-antineutron operators, Phys. Rev. D 93, 016005 (2016).

[23] E. Rinaldi, S. Syritsyn, M. L. Wagman, M. I. Buchoff, C. Schroeder, and J. Wasem, companion paper, Lattice QCD determination of neutron-antineutron matrix elements with physical quark masses, Phys. Rev. D 99, 074510 (2019).

[24] T. Blum et al. (RBC and UKQCD Collaborations), Domain wall QCD with physical quark masses, Phys. Rev. D 93, 074505 (2016).

[25] J. Bijnens and E. Kofoed, Chiral perturbation theory for neutron-antineutron oscillations, Eur. Phys. J. C 77, 867 (2017).

[26] S. Syritsyn, T. Izubuchi, and H. Ohki, Calculation of nucleon electric dipole moments induced by quark chromo-electric dipole moments and the QCD $\theta$-term, arXiv:1901.05455.

[27] E. Shintani, R. Arthur, T. Blum, T. Izubuchi, C. Jung, and C. Lehner, Covariant approximation averaging, Phys. Rev. D 91, 114511 (2015).

[28] T. Doi and M. G. Endres, Unified contraction algorithm for multi-baryon correlators on the lattice, Comput. Phys. Commun. 184, 117 (2013).

[29] W. Detmold and K. Orginos, Nuclear correlation functions in lattice QCD, Phys. Rev. D 87, 114512 (2013).

[30] O. Ledoit and M. Wolf, A well-conditioned estimator for large-dimensional covariance matrices, J. Multivariate Anal. 88, 365 (2004).
[31] G. Golub and V. Pereyra, Separable nonlinear least squares: The variable projection method and its applications, Inverse Probl. 19, R1 (2003); B. W. Rust and D. P. O'Leary, Variable projection for nonlinear least squares problems, Comput. Optim. Applic. 54, 579 (2013).

[32] G. Martinelli, C. Pittori, C. T. Sachrajda, M. Testa, and A. Vladikas, A general method for nonperturbative renormalization of lattice operators, Nucl. Phys. B445, 81 (1995).

[33] B. Blossier, M. Brinet, P. Guichon, V. Morénas, O. Pène, J. Rodríguez-Quintero, and S. Zafeiropoulos (ETM Collaboration), Renormalization of quark propagator, vertex functions, and twist-2 operators from twisted-mass lattice QCD at $N_{f}=4$, Phys. Rev. D 91, 114507 (2015).

[34] S. Bethke, The 2009 world average of alpha(s), Eur. Phys. J. C 64, 689 (2009).

[35] R. N. Mohapatra and R. E. Marshak, Local B-L symmetry of electroweak interactions, Majorana neutrinos and neutron oscillations, Phys. Rev. Lett. 44, 1316 (1980).

[36] K. S. Babu, P. S. Bhupal Dev, and R. N. Mohapatra, Neutrino mass hierarchy, neutron-anti-neutron oscillation from baryogenesis, Phys. Rev. D 79, 015017 (2009).

[37] K. S. Babu, P. S. Bhupal Dev, Elaine C. F. S. Fortes, and R. N. Mohapatra, Post-sphaleron baryogenesis and an upper limit on the neutron-antineutron oscillation time, Phys. Rev. D 87, 115019 (2013). 